\title{
Thyroid Uptake Exceeding 100\%: How Is That Possible?
}

Kathy S. Thomas, Ann Marie Alessi, and Art Hall

M most basic procedures performed in nuclear medicine today. The uptake procedure assesses iodide trapping and organification within the gland (1). It is most commonly performed to differentiate thyroiditis from other forms of thyrotoxicosis and determine the therapeutic dose activity for hyperthyroidism or ablative therapy $(2,3)$.

${ }^{131} \mathrm{I}$ - and ${ }^{123} \mathrm{I}$-sodium iodide are the most commonly used radiopharmaceuticals for thyroid uptake determination. The assessment of the thyroid gland to take up and retain radioactive iodine is based on a ratio of the radioactive capsule (considered to be 100\%) and the amount retained by the gland within a specified time period.

\section{THE ISSUE: IMPORTANCE AND EFFECT}

Thyroid uptake exceeding 100\% seems mathematically impossible. How can the thyroid gland take up more activity than was administered? In most cases, the findings can be traced to specific technical issues that can be easily overlooked in a busy working environment. Inaccurate uptake measurements provide the clinician with erroneous results that directly affect treatment and follow-up patient care.

\section{TECHNICAL ISSUES TO WATCH OUT FOR}

\section{Probe Placement}

- Probe technology is affected by numerous factors including external radiation sources, power, and temperature. - External radiation sources include radiation from diagnostic imaging equipment (x-ray, CT, etc.), radioactive patients, and radioactive contamination. Although walls may be shielded, in most cases it does not include ceiling space where electrical and AC ducts share space and scattered radiation can travel. Radioactive patients from adjacent injection or imaging rooms, residual radiation from previous procedures, and radioactive contamination can increase count rate and affect final results. Remember: millicuries trump microcuries every time!

- Electrical voltage and temperature can also play a role in the accurate detection of energy pulses emitted by a radioactive source. Probe technology should be placed in a room protected from extreme temperature or humidity shifts and should always have a clean power supply.

COPYRIGHT @ 2019 by the Society of Nuclear Medicine and Molecular Imaging.

\section{Counting Technique}

- Geometry (patient and capsule distance) plays a critical role in accuracy. Counting is performed at a distance of $25-35 \mathrm{~cm}$ (activity-dependent) from the face of the probe's collimator. Technologists using a slightly varied positioning technique or distance can be the difference between an accurate uptake and one with suggestive findings (count rate too high/low). Suggestion: to test the accuracy and reproducibility of the counting technique, perform a thyroid uptake by counting the capsule as the standard, then using that same capsule for the 2- to 6-h and 24-h uptake. All results should be within $\pm 3 \%$ of $100 \%$ for every counting period for every technologist. If not, make it a quality project in your department to develop a reproducible technique to ensure that initial full capsule counts as well as follow-up patient counts are consistent and accurate. The technique should include correct centering, distance, and positioning of the probe, thyroid phantom, and patient. Important: the probe

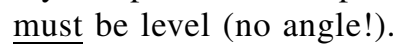

\section{Not All Capsules or Liquid Standards Are Equal}

- Assuming that all capsules or liquid doses in the same lot have exactly the same count rate may be a source of error. Although the ratio calculation (patient counts/ standard counts) is not extremely sensitive and small variations in count rate will not affect the final results, it is important to realize that when using a single standard for all procedures from the same lot may introduce an error factor. If a patient is suspected of having an extremely high uptake ( $>75 \%-80 \%)$, the difference between actual capsule counts for the dose administered versus the dose used as the standard may be the difference between an uptake exceeding $100 \%$ or one that is more appropriately below $100 \%$.

\section{Adequate Counting Time}

- A short counting time can invalidate uptake results. Counting time should be set at a minimum of $60 \mathrm{~s}$.

\section{Attenuating Materials}

- The attenuation properties of the acrylic or graphite distance/measurement rod used on probe technology may reduce total counts by $13.1 \%$ and $10 \%$, respectively. The assumption that counting with the measurement rod in place for both the capsule count and the patient count will cancel out any error factor attributed to attenuation is incorrect. The measurement rod and 
LED centering device should always be moved out of the field of view before counting is initiated. Additionally, incorrect placement of the capsule in the pill holder of the thyroid phantom (thick side forward) will also result in increased attenuation and a reduction of total counts.

\section{Basic Quality Issues}

- Not to be overlooked, daily, weekly, quarterly, and annual quality control procedures ensure operational accuracy of probe technology.

\section{BOTTOM LINE}

Although thyroid uptake measurements are not as technologically challenging as many of the other procedures performed in nuclear medicine, the accuracy of the final result and patient outcome relies on the constancy, accuracy, and reproducibility of the nuclear medicine technologist performing the procedure.

\section{REFERENCES}

1. Bolus NE, Glasgow KW. Review of Nuclear Medicine Technology: Preparation for Certification Examinations. 5th ed. Reston, VA: Society of Nuclear Medicine and Molecular Imaging; 2018.

2. ACR-SNM-SPR practice guideline for the performance of thyroid scintigraphy and uptake measurements. Revised 2009, resolution 17. Society of Nuclear Medicine and Molecular Imaging website. http://snmmi.files.cms-plus.com/docs/Thyroid Scintigraphy_1382732120053_10.pdf. Accessed July 22, 2019.

3. Farrell MB, Mantel ES, Basso DA, et al. Thyroid imaging and uptake. In: Quick Reference Protocol Manual. Reston, VA: Society of Nuclear Medicine and Molecular Imaging; 2014:149-154. 